\title{
Pathological Breathing Patterns After Pneumococcal Rhombencephalitis
}

\author{
Sophie Muyldermans MD, Bart Vrijsen PT MSc, Marc Decramer MD PhD, \\ Catharina Belge MD PhD, Dries Testelmans MD PhD, and Bertien Buyse MD PhD
}

\section{Introduction}

Apneustic breathing is an abnormal breathing pattern characterized by a prolonged inspiratory time with an endinspiratory pause versus a shorter expiratory time. ${ }^{1}$ The termination of respiration is considered to be controlled by the pontine respiratory group (PRG). The apneustic breathing pattern is very rare in humans. Patients with autonomic breathing failure have an impaired autonomic control of ventilation, although their voluntary control remains intact. $^{2}$ This is usually because of congenital central hypoventilation syndrome. In rare cases, it can be observed following brainstem lesions. Because of the very rare descriptions of these pathological breathing patterns, still little is known about the impact of specific brainstem lesions on the control of ventilation in humans.

We present a case of a patient with an incomplete locked-in syndrome and a respiratory drive dysfunction consisting of the combination of an apneustic-like breathing pattern while awake and an autonomic breathing failure while asleep due to the uncommon cause of pneumococcal rhombencephalitis.

\section{Case Summary}

Two years ago, a 15-y-old girl was diagnosed with pneumococcal rhombencephalitis. The diagnosis was made based on a positive polymerase chain reaction for Streptococcus pneumoniae from cerebrospinal fluid and on typ-

All authors are affiliated with the Department of Pulmonary and Sleep Medicine, University Hospitals Leuven, Leuven, Belgium. Dr Muyldermans is also affiliated with the Department of Pulmonology, AZ Sint-Jan Brugge-Oostende AV, Brugge, Belgium.

The authors have disclosed no conflicts of interest.

Correspondence: Sophie Muyldermans MD, Department of Pulmonology, AZ Sint-Jan Brugge-Oostende, Ruddershove 10, B-8000 Bruges, Belgium. E-mail: sophie.muyldermans@azsintjan.be.

DOI: $10.4187 /$ respcare. 02510 ical abnormalities of the cranial computed tomography (narrow basal cisterns in the fossa posterior and a blurred delineation of the brainstem). After 3 weeks and several weaning trials, it became evident that she remained ventilator-dependent, and a tracheostomy was performed. She was discharged 3 months after initial presentation on nearly continuous tracheal ventilation in pressure assist-control mode. At the time of evaluation, she was quadriplegic except for some left-hand mobility.

A recent neurological investigation in the Coma Science Group of Liège, Belgium, revealed the following data during magnetic resonance imaging (MRI): cerebellar damage and severe brainstem atrophy, most prominent in the medulla oblongata, with areas of chronic gliosis with Wallerian degeneration at the level of the pons and medulla oblongata (Fig. 1). Although positron emission tomography also revealed large hypometabolic regions in the cortex, standardized cognitive testing revealed no cognitive dysfunction. She was diagnosed with an incomplete locked-in syndrome with atypical additional supratentorial lesions.

The girl was ventilated nearly full-time by tracheal ventilation. However, she was able to remain free from the ventilator for a maximum of $2 \mathrm{~h}$ when someone was talking to her. The patient was referred to our department for implantation of a phrenic nerve pacing system to increase ventilator-free breathing. A series of respiratory tests were performed, and prior to each test, the patient and her father, who was always present, were informed about the test and agreed to the procedure.

We first performed a phrenic nerve conduction study according to the method described by MacLean and Mittoni. ${ }^{3}$ This study showed a normal conductance with an asymmetrical diaphragmatic compound muscle action potential. Fluoroscopy of the diaphragm during stimulation showed adequate movement of the diaphragm, although less pronounced on the left side. This unilateral mild reduction in muscle action potential clearly did not explain her ventilator dependence.

For this reason, we performed further investigation. We switched off the tracheal ventilation during the daytime 

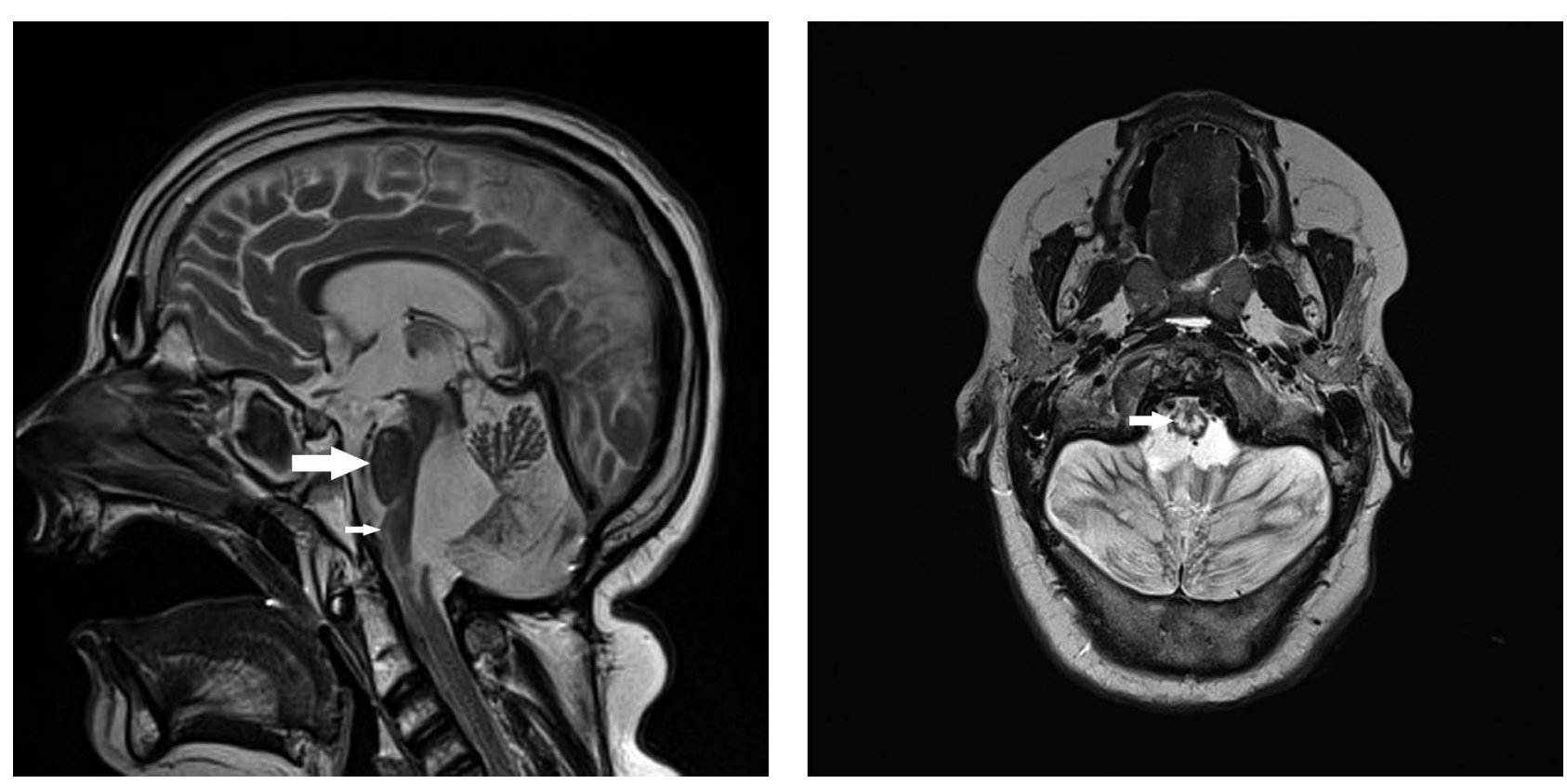

Fig. 1. Cerebellar damage and brainstem atrophy with areas of chronic gliosis with Wallerian degeneration at the level of the pons and medulla oblongata. Left: Sagittal T2-weighted MRI image. Pons (large arrow); medulla oblongata (small arrow). Right: Axial T2-weighted MRI image, medulla oblongata (arrow).

while the patient was fully awake, which revealed a deep and slow breathing pattern at a rate of 8 breaths/min with apneustic characteristics (Fig. 2). Over a period of $\sim 1 \mathrm{~h}$, a gradual increase in cardiac rate (from 75 to 85 beats $/ \mathrm{min}$ ) was observed. We did not have the arterial blood gas data for the patient, but a validated transcutaneous carbon dioxide monitor (Tosca 500, Radiometer, Brønshøj, Denmark $)^{4}$ showed an elevation of transcutaneous $\mathrm{P}_{\mathrm{CO}_{2}}\left(\mathrm{P}_{\mathrm{tcCO}}\right.$; from 38 to $45 \mathrm{~mm} \mathrm{Hg}$ ) (Fig. 3). Figure 3 shows that oxygen saturation was relatively low, although the patient was ventilated. This was because of mucus accumulation and frequent need for suctioning.

When the ventilator was switched off during slow wave sleep, the patient failed to breathe at all, although she was not overventilated (Fig. 4). After $20 \mathrm{~s}$, oxygen desaturation occurred, and because of severe hypoxia, we restarted the ventilator after $38 \mathrm{~s}$.

\section{Discussion}

This case shows that an infectious rhombencephalitis with visible lesions at different levels of the brainstem can cause a combination of pathological breathing patterns. This patient showed a respiratory drive dysfunction with an apneustic-like breathing pattern with intermittent oxygen desaturations and an increase in $\mathrm{P}_{\mathrm{tcCO}}$ while awake and autonomic breathing failure while asleep.

The term rhombencephalitis is used to describe encephalitis involving the brainstem and/or cerebellum. Pneumo- coccal infection is a very rare cause of rhombencephalitis, with only a few cases reported previously. ${ }^{5}$

Patients with locked-in syndrome are quadriplegic and have anarthria with preserved consciousness and respiratory, cardiac, and vasomotor functions. Locked-in syndrome is caused by destructive bilateral brainstem lesions, affecting the corticospinal, corticopontine, and corticobulbar tracts. The most common causes are ischemic stroke and hemorrhage. ${ }^{6,7}$ Other causes include trauma, ${ }^{7}$ pontine abscess, ${ }^{8}$ and brainstem tumors. ${ }^{9}$ Our patient suffers from an incomplete locked-in syndrome, with preservation of voluntary breathing and some left-hand movement and mouth opening. An MRI confirmed lesions in the medullary pyramids and the pons (see Fig. 1), which are responsible for the locked-in status and the described disorders. To our knowledge, this is the first case of an (incomplete) locked-in syndrome due to pneumococcal rhombencephalitis. Moreover, because of a broader lesion of the lateral tegmentum of the pons, the patient had autonomic breathing failure, which is not typical for locked-in syndrome.

Control of breathing in humans is complex and is not yet fully understood. It is not our goal to discuss this in detail, but we will provide a short overview. In normal subjects, respiration is controlled by respiratory centers in the brainstem. ${ }^{10}$ These respiratory centers receive input from central respiratory pacer cells, central and peripheral chemoreceptors, mechanoreceptors, the midbrain, and volitional pathways (voluntary respiration ${ }^{11}$ ). Three brainstem areas are considered important: the PRG or pneumo- 

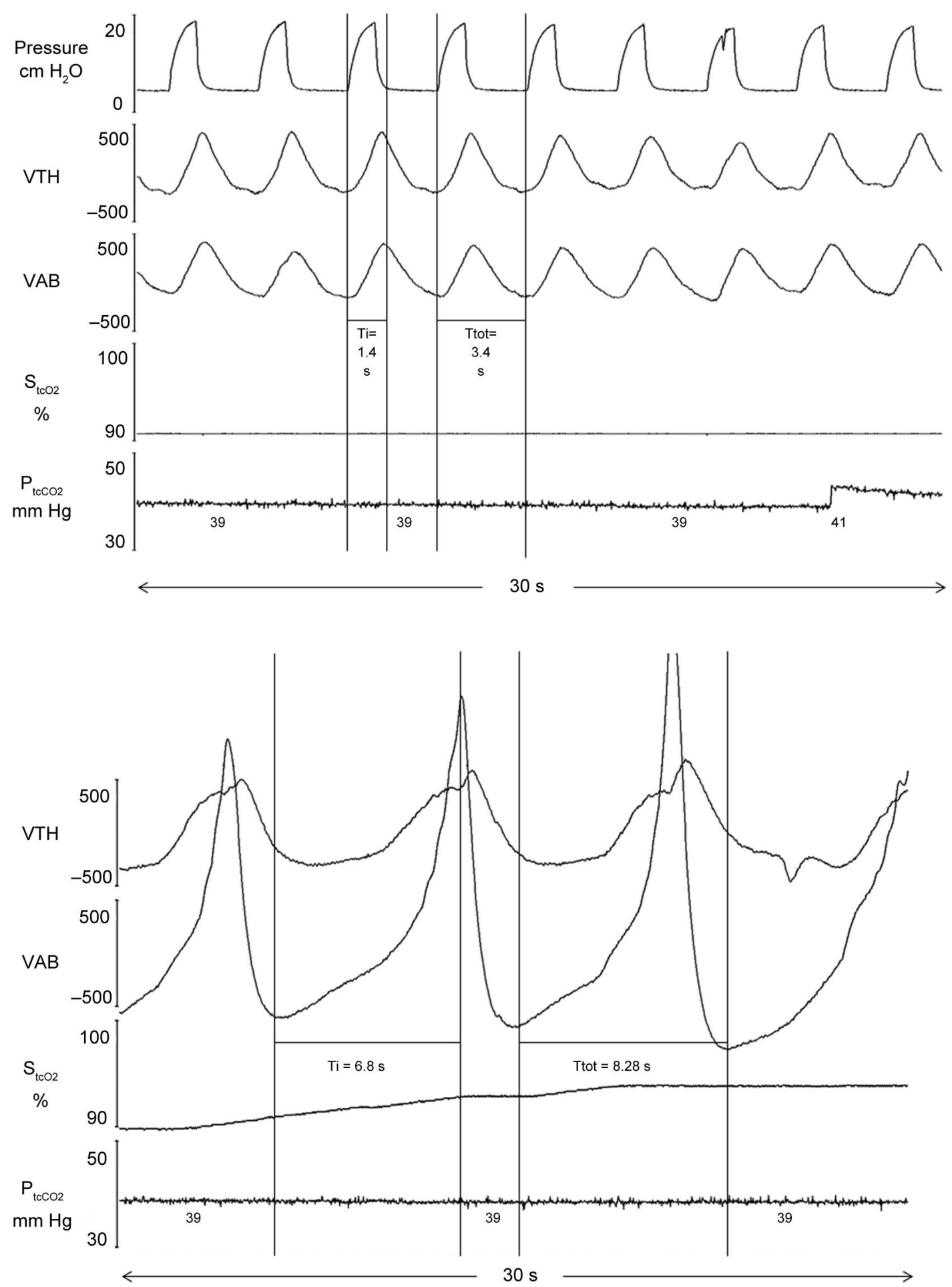

Fig. 2. Breathing pattern while awake. Top: Polysomnography extract of $30 \mathrm{~s}$ while the patient was awake and ventilated. Breathing frequency 18 breaths/min, inspiratory time $1.4 \mathrm{~s}$, total respiration time $3.4 \mathrm{~s}$; as set on the pressure-controlled mode of the ventilator. Bottom: Extract of $30 \mathrm{~s}$ while the patient was awake and not ventilated. Breathing frequency 8 breaths/min, inspiratory time $6.8 \mathrm{~s}$, total respiration time $8.2 \mathrm{~s}$; representation of the apneustic-like breathing pattern. VTH $=$ movement of thorax, VAB $=$ movement of abdomen, $\mathrm{S}_{\mathrm{tcO}_{2}}=$ transcutaneous oxygen saturation, $\mathrm{P}_{\mathrm{tcCO}_{2}}=$ transcutaneous $\mathrm{P}_{\mathrm{CO}_{2}}$.

taxic center in the pons and the ventral (VRG) and dorsal (DRG) respiratory groups in the medulla. The PRG con- tains inspiratory, expiratory, and phase-spanning neurons and is involved in the modification and fine control of the 


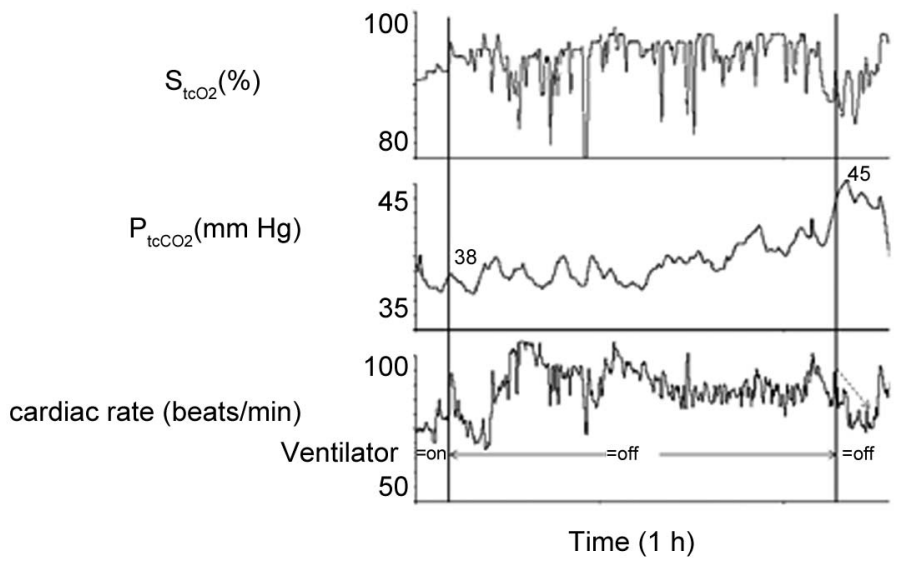

Fig. 3. Measurement of transcutaneous oxygen saturation $\left(\mathrm{S}_{\mathrm{tcO}_{2}}\right)$, transcutaneous $\mathrm{P}_{\mathrm{CO}_{2}}\left(\mathrm{P}_{\mathrm{tcCO}}\right)$, and cardiac rate while the patient was awake and the ventilator was switched off during $1 \mathrm{~h} . \mathrm{S}_{\mathrm{tcO}_{2}}$ remained stable. $\mathrm{P}_{\mathrm{tcCO}}$ increased from 38 to $45 \mathrm{~mm} \mathrm{Hg}$. Cardiac rate increased from 75 to 85 beats $/ \mathrm{min}$.

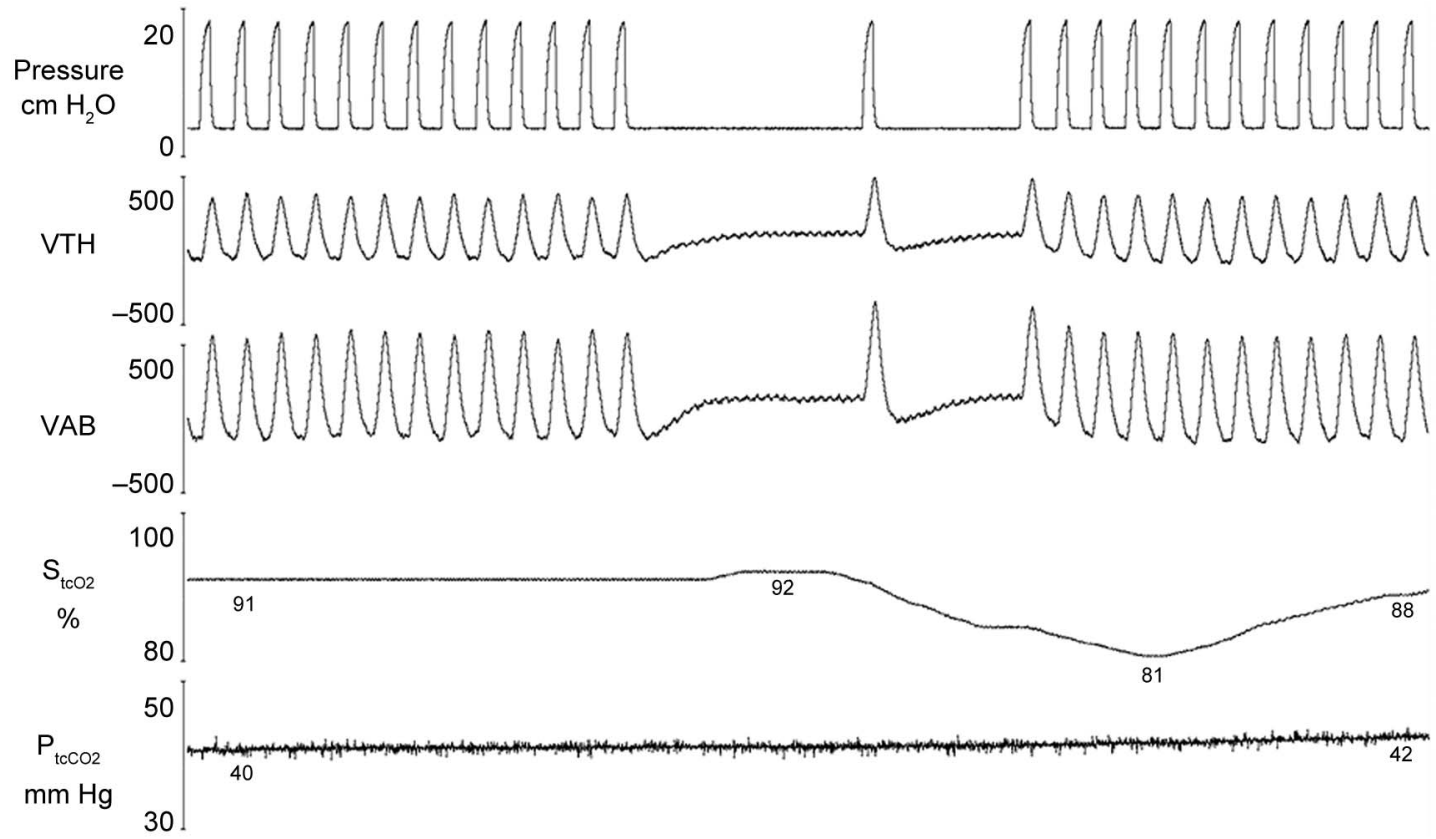

Fig. 4. Breathing pattern while asleep. Polysomnography extract of 2 min while the patient was in deep (slow wave) sleep. On the ventilator in pressure assist-control mode, the timed frequency was abruptly diminished from 18 to 1 breaths/min for a duration of $38 \mathrm{~s}$. Although not overventilated (transcutaneous oxygen saturation $\left[\mathrm{S}_{\mathrm{tcO}_{2}}\right] 91 \%$ and transcutaneous $\mathrm{P}_{\mathrm{CO}_{2}}\left[\mathrm{P}_{\mathrm{tcCO}}\right] 41 \mathrm{~mm} \mathrm{Hg}$ ), the patient failed to breathe autonomously while asleep, and $\mathrm{S}_{\mathrm{tcO}_{2}}$ decreased to $81 \% . \mathrm{P}_{\mathrm{tcCO}_{2}}$ remained stable due to the longer averaging time $(50 \mathrm{~s})$ of $\mathrm{P}_{\mathrm{tccO}}$ measurement. $\mathrm{VTH}=$ movement of thorax, $\mathrm{VAB}=$ movement of abdomen.

respiratory rhythm. The VRG is the generator of the respiratory rhythm, whereas the DRG is the primary rhythmic respiratory drive to phrenic motor neurons.

Brainstem lesions may cause characteristic abnormalities in breathing pattern. In animals, apneustic breathing is produced by pontine section. This type of breathing consists of a prolonged inspiratory time with an end-inspiratory pause versus a shorter expiratory time and is very rare in humans. ${ }^{1}$ Our patient had an apneustic-like breathing pattern while awake, with a deep and prolonged inspira- tion, and a relative longer inspiration time versus a shorter expiration time but without obvious end-inspiratory pauses (see Fig. 2). This breathing pattern resulted in exhaustion, with an increase in $\mathrm{P}_{\mathrm{tcCO}}$ and cardiac rate after a short time of switching off the ventilator (see Fig. 3). On MRI, chronic gliosis with Wallerian degeneration at the level of the pons could be seen (Fig. 1) and is probably responsible for this apneustic-like breathing pattern.

Autonomic breathing failure is caused by destruction of the lateral medulla affecting the VRG, its connections with 


\section{Pathological Breathing Patterns After Pneumococcal Rhombencephalitis}

the DRG, and the fibers crossing over in the reticulospinal tract. This is caused mainly by both bilateral and unilateral medullary infarctions or by bulbar poliomyelitis. ${ }^{2}$ In our patient, the MRI revealed chronic gliosis with Wallerian degeneration at the level of the medulla oblongata (Fig. 1) as a consequence of pneumococcal rhombencephalitis, which could explain the central origin of the apnea. To our knowledge, this is the first description of autonomic breathing failure caused by pneumococcal rhombencephalitis.

Because the apneustic-like breathing pattern resulted in exhaustion and an increase in $\mathrm{P}_{\mathrm{tcCO}}$ a short time after switching off the ventilator, implantation of a phrenic nerve pacing system was proposed. Phrenic nerve pacing can improve ventilation and eliminate the need for continuous positive-pressure ventilatory support in selected patients with respiratory insufficiency due to injury or disease of the central nervous system. It increases ventilator-free breathing and reduces costs. Restoring negative-pressure ventilation also improves olfaction. Possible complications of this treatment are related to the surgery (local infection and pulmonary complications after thoracic surgery) or to the pacing system (technical malfunction or injury to the phrenic nerve). ${ }^{12}$ Because of her incurable locked-in status, the patient declined this therapy.

\section{Teaching Points}

- Pneumococcal infection can involve the brainstem and/or cerebellum, causing rhombencephalitis.

- Failure to wean a patient from the ventilator is not always a problem of respiratory muscle weakness. Persistent brainstem damage should be considered in the differential diagnosis.

- Pneumococcal rhombencephalitis can cause incomplete locked-in syndrome, as well as brainstem lesions with specific pathological breathing patterns.

- The pneumotaxic center in the pons is involved in respiratory rhythm control. Brainstem lesions at the level of the pons create an apneustic-like breathing pattern with a deep and prolonged inspiration.

- The medulla contains the VRG and DRG. Destruction of the lateral medulla causes autonomic breathing failure.

- In patients with impaired breathing due to a central nervous system lesion, phrenic nerve pacing could be considered as a treatment option to improve ventilator-free breathing.

\section{REFERENCES}

1. Plum F, Alvord EC Jr. Apneustic breathing in man. Arch Neurol 1964;10(1):101-112.

2. Severinghaus JW, Mitchell RA. Ondine's curse: failure of respiratory center automaticity while awake. Clin Res 1962;10(1):122.

3. MacLean IC, Mittioni TA. Phrenic nerve conduction studies: a new technique and its application in quadriplegic patients. Arch Phys Med Rehabil 1981;62(2):70-73.

4. Rafiq MK, Bradburn M, Proctor AR, Billings C, Bianchi S, McDermott CJ, Shaw PJ. Using transcutaneous carbon dioxide monitor (TOSCA 500) to detect respiratory failure in patients with amyotrophic lateral sclerosis: a validation study. Amyotroph Lateral Scler 2012;13(6):528-532.

5. Moragas M, Martínez-Yélamos S, Majós C, Fernández-Viladrich P, Rubio F, Arbizu T. Rhombencephalitis: a series of 97 patients. Medicine 2011;90(4):256-261.

6. Patterson JR, Grabois M. Locked-in syndrome: a review of 139 cases. Stroke 1986;17(4):758-764.

7. León-Carrión J, van Eeckhout P, Domínguez-Morales Mdel R, PérezSantamaría FJ. The locked-in syndrome: a syndrome looking for a therapy. Brain Inj 2002;16(7):571-582.

8. Murphy MJ, Brenton DW, Aschenbrener CA, Van Gilder JC. Locked-in syndrome caused by a solitary pontine abscess. J Neurol Neurosurg Psychiatry 1979;42(11):1062-1065.

9. Cherington M, Stears J, Hodges J. Locked-in syndrome caused by a tumor. Neurology 1976;26(2):180-182.

10. Bolton C, Chen R, Wijdicks E, Zifko U. Neurology of breathing. Philadelphia: Butterworth-Heinemann; 2004:26-32, 53-60.

11. Heywood P, Murphy K, Corfield DR, Morrell MJ, Howard RS, Guz A. Control of breathing in man; insights from the 'locked-in' syndrome. Respir Physiol 1996;106(1):13-20.

12. DiMarco AF. Phrenic nerve stimulation in patients with spinal cord injury. Respir Physiol Neurobiol 2009;169(2):200-209. 